\title{
Visualization of a Sweeping Jet by Laser Speckle Retro-reflective Background Oriented Schlieren
}

\author{
Laura K. Kushner* \\ ACI/NASA Ames Research Center, Moffett Field, CA 94035, USA \\ James T. Heineck, ${ }^{\dagger}$ Bruce L. Storms ${ }^{\ddagger}$ and Robert E. Childs $\S$ \\ NASA Ames Research Center, Moffett Field, CA 94035, USA
}

\begin{abstract}
The National Aeronautics and Space Administrations Environmentally Responsible Aviation (ERA) Program is currently investigating the use of sweeping jet actuators as active flow control devices to improve the aerodynamic performances of vertical tails on commercial transporters. Computational Fluid Dynamics (CFD) simulations have shown that the motion of the jet is not a simple sinusoid, but lingers at the extremes of jet deflection. As part of an effort to better understand this non-sinusoidal behavior and validate the CFD, a sweeping jet actuator was tested in the 48-by-32-inch wind tunnel in the Fluid Mechanics Laboratory (FML) at NASA Ames Research Center. The jet was visualized at very high frequencies using a new technique: laser speckle retroreflective background oriented schlieren (RBOS). These measurements confirmed the non-sinusoidal nature of the jet motion. Although measurements were also made by Particle Image Velocimetry (PIV) that resolved the flow velocities in the jet, only the new RBOS technique could provide high enough frequencies to both spatially and temporally resolve the non-sinusoidal motion. This paper presents the laser speckle RBOS method and visualization, as well as a brief comparison to CFD simulations.
\end{abstract}

\section{Introduction}

Background Oriented Schlieren (BOS $)^{1}$ is an optical technique similar to schlieren and shadowgraph that measures the deflection of light rays from an illumination source due to refractive index gradients in fluid flow. It is used in situations where traditional schlieren techniques are not feasible due to optical constraints, as the only optical access that is required is a small window that can accommodate both the camera and the light source. A speckle pattern in the background of the region of interest is imaged in quiescent, wind-off conditions and also at wind-on test conditions. When a density gradient lies in the foreground of a BOS background, it produces a change in the index of refraction that causes the speckles that lie in the background of the density gradient to appear to shift in the direction of the decreasing density. This characteristic makes it possible to precisely locate flow features in BOS images. These apparent shifts in the background are measured in the same way as particle displacements are measured in PIV images: points of interest in the images are defined by a rectangular grid, and the local image-plane displacement at each node of the grid due to density gradients is measured by cross correlating the wind-off and wind-on images in a small (e.g., $20 \times 20$ pixel) interrogation window centered on the node. The displacement of the peak of the correlation function from the center of the interrogation window measures the local displacement between the wind-off and wind-on images. When speckle displacements at all nodes of the grid are displayed as a contour plot, a schlieren-like image is produced. Therefore BOS can provide schlieren-type data in wind tunnels that lack large, expensive, schlieren-quality windows.

\footnotetext{
${ }^{*}$ Research Engineer, ACI/Experimental Aero-Physics Branch, M/S 260-1, Non-Member.

${ }^{\dagger}$ Photographic Technologist, Experimental Aero-Physics Branch, M/S 260-1, Non-Member.

¥Aerospace Engineer, Experimental Aero-Physics Branch, M/S 260-1, Non-Member.

$\S$ Aerospace Engineer, STC/Systems Analysis Branch M/S 258-1, Non-Member.
} 
With Retroreflective Background Oriented Schlieren (RBOS), ${ }^{2}$ dark speckles are applied to a retroreflective background and the illumination source is placed with the camera, maximizing the retro-reflected light returned from the background. This improves the BOS technique in two ways. First, it increases the contrast between the dark speckles and bright background, improving the signal-to-noise ratio (SNR), which allows shorter integration times that can freeze flow unsteadiness. Second, it allows a smaller lens aperture and thus greater depth-of-field. For a camera focused on the background, this improves the focus in the region of interest and allows smaller flow features to be resolved.

Laser speckle RBOS ${ }^{3}$ takes the process one step further - instead of painting or printing speckles on the retroreflective background, a laser beam is expanded and aligned and made collinear to the camera field of view through the use of a beam-combining optic. The laser beam then passes through the region of interest and is reflected off of a retroreflective background. The uneven surface of the retroreflector sets up constructive and destructive interference on the reflected beam, which makes the background appear as if it were speckled, instead of evenly illuminated. This speckle is ideal for the cross-correlation algorithms, as the peaks are gaussian, whereas the speckles from an application of paint are not.

\section{Sweeping Jet Actuator Test Setup in the FML}

Sweeping jet actuators are currently being studied as a method of active flow control to improve the performance of wings and tails. The sweeping jet actuator blows compressed air over the rudder and injects energy into the flow, thus keeping the flow attached at higher rudder-deflection angles. Typically, rudders are sized for single engine failure, leaving them oversized for nominal flight conditions. If sweeping jet actuators can improve the efficiency of the tail, vertical tail sizes could be reduced, leading to lighter, more fuel-efficient structures.

The sweeping jet actuator itself is a plenum with an entrance and an exit nozzle, and encircled by two feedback paths. When air is forced into the cavity, it initially follows one feedback path. "A backflow then develops in the feedback loop and forces the jet flow to detach from that surface and attach to the opposite surface. When the flow attaches to the opposite surface, a backflow is developed in the

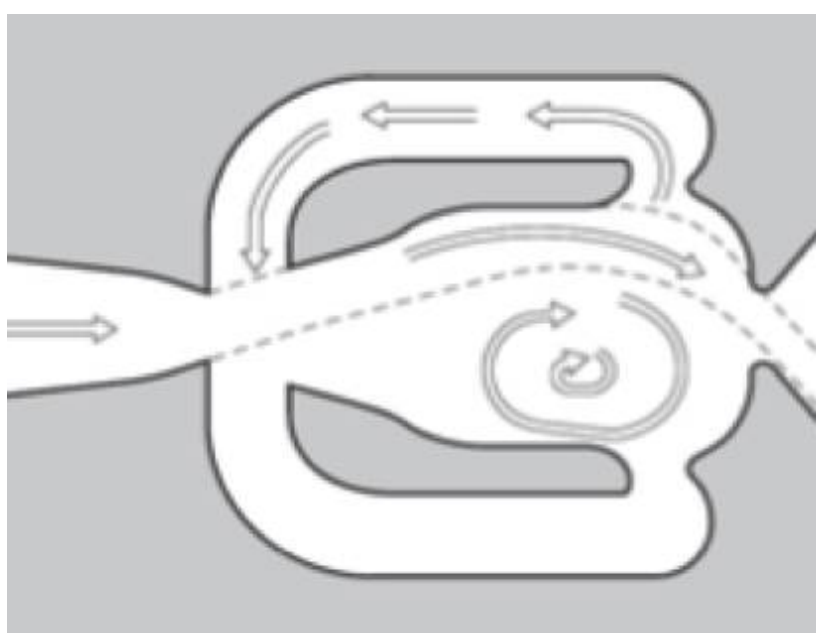

Figure 1. Example of a sweeping jet actuator geometry, from Koklu and Melton. ${ }^{4}$ other feedback loop which forces the jet to switch back to its initial state. The process then repeats itself, thus producing a self-sustaining oscillation." ${ }^{4}$ Figure 1 shows an example of the geometry.

\section{A. Wind Tunnel}

The test was conducted in the FML 48-inch by 32-inch Indraft Subsonic Wind Tunnel (Test Cell 2). This tunnel is an open circuit indraft tunnel with a sonic throat. The test section is 48 inches wide, 32 inches tall, and approximately 120 inches long. The maximum flow rate with the sonic throat clamshells wide open is $108,800 \mathrm{cu} \mathrm{ft} / \mathrm{min}$, which produces a maximum velocity of approximately $170 \mathrm{fps}(50 \mathrm{~m} / \mathrm{s})$, and corresponds to a dynamic pressure of approximately 34 psf, and a Reynolds Number of approximately 1 million per foot. The tunnel has a free stream turbulence level of below $0.1 \%$. Though the maximum empty tunnel wind speed is approximately $50 \mathrm{~m} / \mathrm{s}$ (164 fps), the maximum tunnel speed was limited so that the pressure difference across the test section windows was under 0.25 psi.

Facility operating instrumentation includes pressure, temperature, and humidity transducers to quantify tunnel speed and dynamic pressure. For this test, additional instrumentation included Kulites in the model, a PIV system, and the RBOS system. In order to accommodate the laser required for the PIV measurements, the test section's plexiglass sidewalls were replaced with new sidewalls made of wood with optical quality glass windows. 


\section{B. Sweeping Jet Actuator Model}

The sweeping jet actuator and support hardware were made from T6160 Aluminum. Two actuators inlaid in airfoils were made - one for PIV with a window in the center of the wing (Figure 2), and another without the window, but with additional pressure sensors near the actuator exit. The test article was mounted parallel to the free stream flow in the wind tunnel so as to minimize edge-effects due to lift. A splitter plate was also included at the downstream end of the model to minimize flow unsteadiness due to Strouhal shedding. Air flow to the actuator was supplied by a reciprocating air compressor. For the RBOS portion of the test, the actuator and airfoil with the window was used.

The model was also equipped with miniature Kulite pressure transducers to measure surface pressures both in the actuator plenum and downstream of

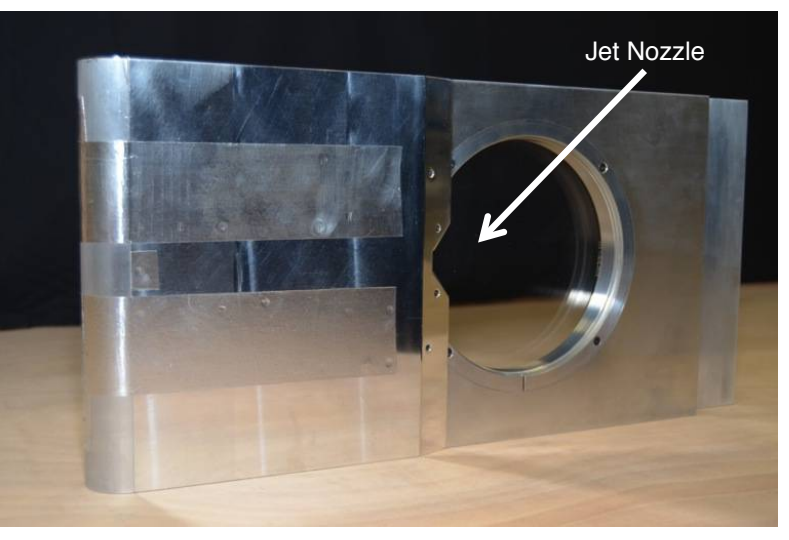

Figure 2. Sweeping Jet Actuator Model the nozzle throat.

\section{RBOS System}

The Laser RBOS system used a Quantronix Darwin Duo Nd:YLF $527 \mathrm{~nm}$ Class 4 laser. The laser operated at 2, 4, 8 and $20 \mathrm{kHz}$ with $25 \mathrm{~mJ} /$ pulse. $20 \mathrm{kHz}$ was achieved by operating the two internal lasers at $10 \mathrm{kHz}$ each, with $50 \mu$ s delay introduced to one. Each laser plusle was approximately $160 \mathrm{~ns}$ in duration and the power at this high frequency dropped to $5 \mathrm{~mJ} /$ pulse.

Two cameras were used: a Phantom 641 high-speed camera typically used for PIV, and a Photron Fastcam SA-Z. The Phantom 641 has a rectangular sensor $(2560 \mathrm{x} 1600)$ with a $10 \mu \mathrm{m}$ pixel pitch and maximum full-frame speed of $1450 \mathrm{fps}$. The Photron Fastcam SA-Z has a square sensor (1024 x 1024) with $20 \mu \mathrm{m}$ pixel pitch and maximum full-frame speed of 20,000 fps.

The laser was located on the side of the wind tunnel and the beam was expanded outside the wind tunnel and reflected to a beam combiner in front of the camera lens. The reflected beam was directed through a window in the side wall of the wind tunnel to the window in the airfoil at the sweeping jet actuator nozzle exit. The transmitted beam was directed into a beam dump. Once the reflected beam passed through the airfoil window, it was reflected again off a 3M Scotchlite 900x retroreflective background that was adhered to an aluminum plate on the far tunnel side wall, back through the airfoil window and transmitted through the beam splitter and into the camera lens. Due to the window geometry of the wind tunnel and the turntable the airfoil could not be mounted so that the camera could look directly though the tunnel window to the window in the airfoil. A schematic of the RBOS system is shown in Figure 3.

During this test, the Phantom 641 was windowed to various sizes and data were acquired at 2, 4, and 8 $\mathrm{kHz}$ for four different sweeping jet actuator pressures, at both wind-on and wind-off conditions. Using the Photron SA-Z, data were acquired at $20 \mathrm{kHz}$ for one sweeping jet actuator pressure at wind-off conditions. Table 1 summarizes the test conditions. Laser RBOS data were acquired separately from the PIV and Kulite data. The laser was synced to the frame sync of the camera, so that the laser pulse fell within the exposure time of the image. Data acquisition was manual, at the onset of flow from the sweeping jet actuator. The bulk of the data were processed post-test, however, a few frames were processed as quick checks during acquisition. Examples of the unprocessed data are shown in Figure 4.

Table 1. Test Condition Summary

\begin{tabular}{|c|c|c|}
\hline RBOS data rate $(\mathrm{kHz})$ & Sweeping Jet Pressure (psig) & Tunnel Speed $(\mathrm{m} / \mathrm{s})$ \\
\hline 2 & $6,13,25,38$ & 0,56 \\
4 & $6,13,25,38$ & 0,56 \\
8 & $6,13,25,38$ & 0,56 \\
20 & 38 & 0 \\
\hline
\end{tabular}




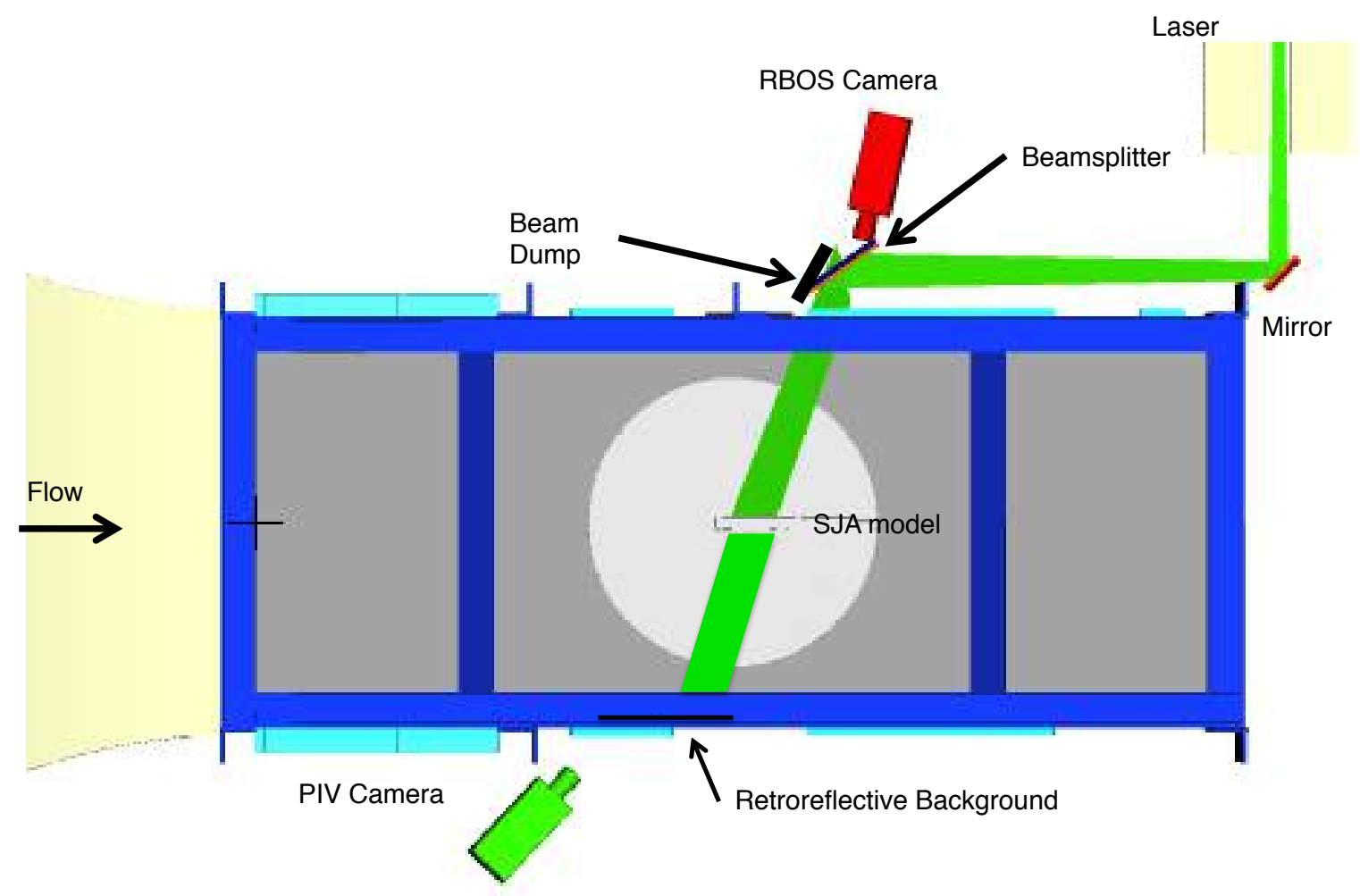

Figure 3. Top view schematic of the RBOS system. Tunnel structure is show in navy; tunnel windows in cyan. The RBOS camera is red, while the PIV camera is green. The path of the expanding laser beam is traced in green, the beamsplitter is orange and the beam dump and retroreflective background are black. Flow of both the wind tunnel and sweeping jet actuator is from left to right. 


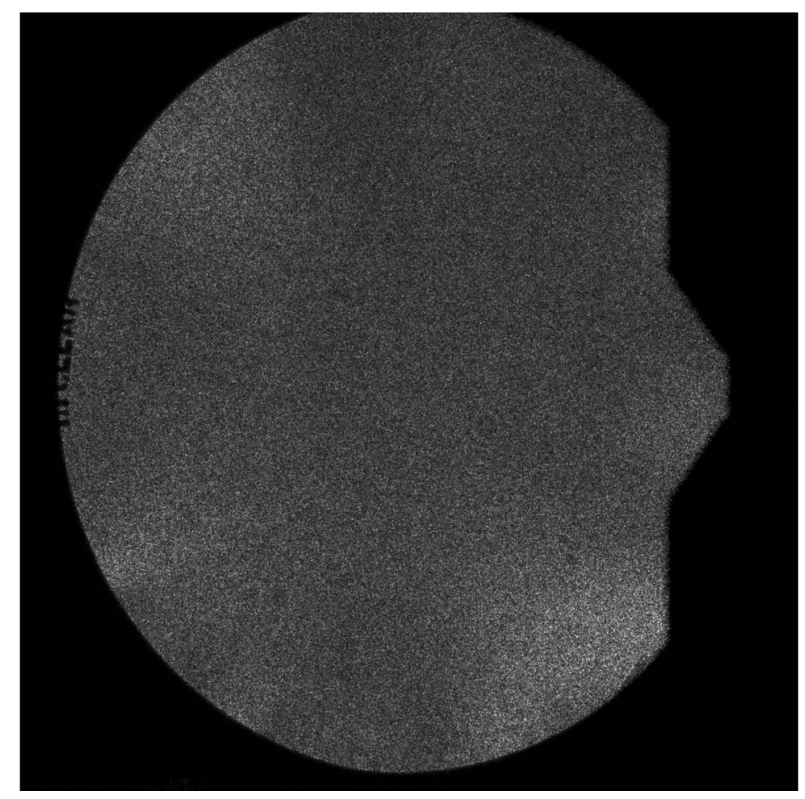

(a) $2 \mathrm{kHz}$ - full FOV, Phantom 641

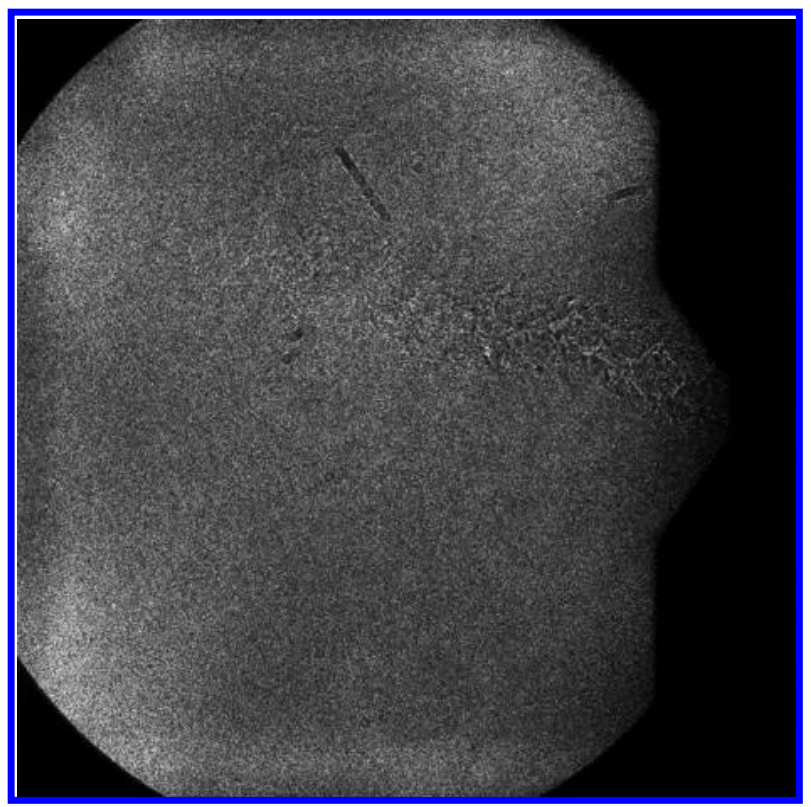

(c) $20 \mathrm{kHz}$ - full FOV, Photron SA-Z

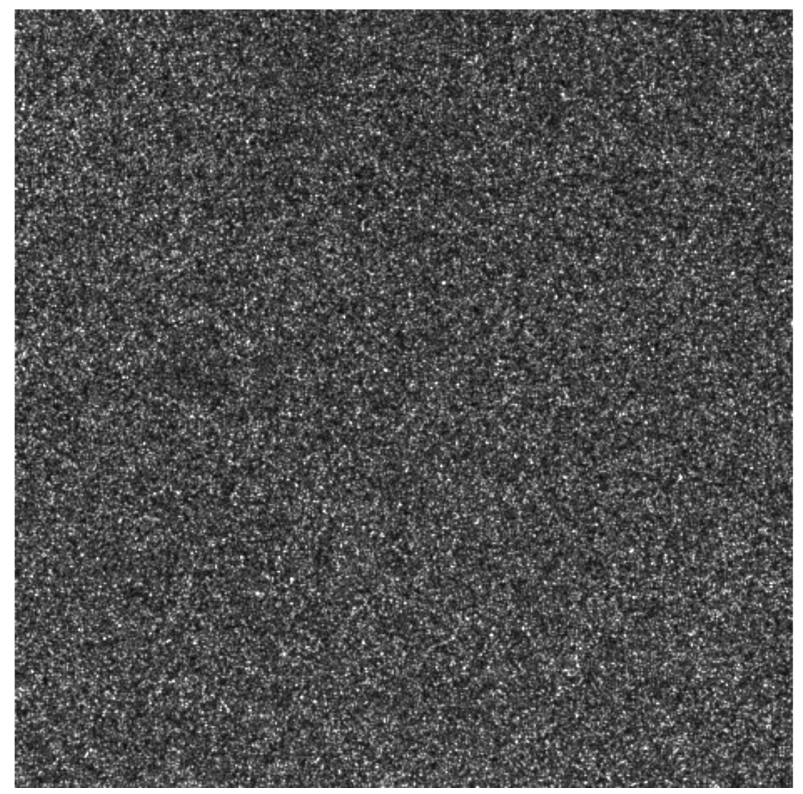

(b) $2 \mathrm{kHz}$ - close-up, Phantom 641

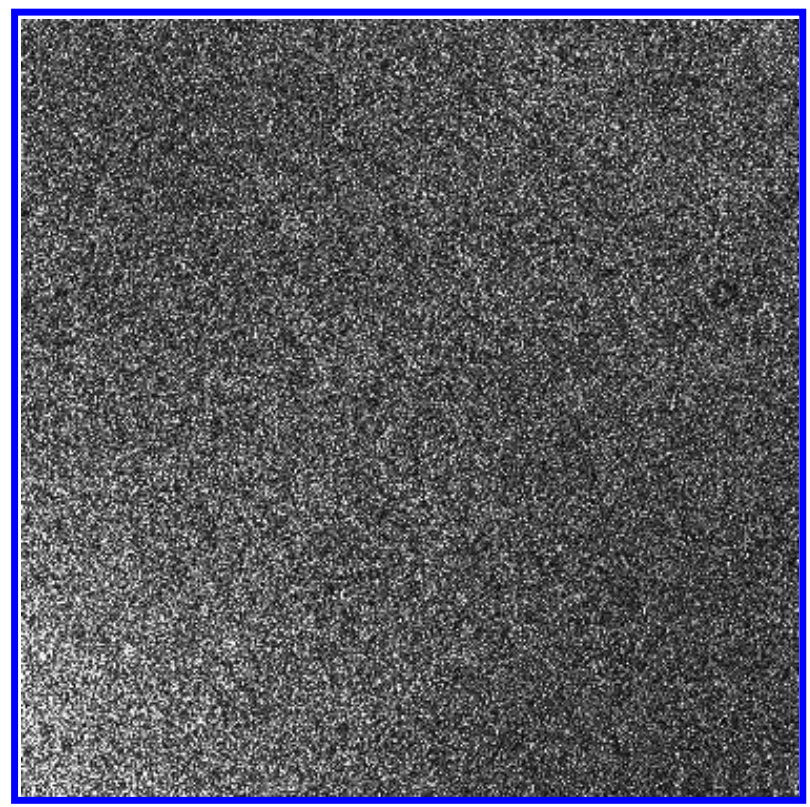

(d) $20 \mathrm{kHz}$ - close-up, Photron SA-Z

Figure 4. Background Speckle Examples

\section{Data}

The data were processed using a commercial PIV software package, PIVView, ${ }^{5}$ in speckle displacement mode. Each processed RBOS image shows the contour plot of the background speckle shift in either the horizontal direction, which corresponds to the horizontal density gradient, or in the vertical direction, which corresponds to the vertical density gradient. An example of the processed data is shown in Figures 5 and 6 for a wind-off case. Note the Mach-wave radiation visible in Figure 5a., as well as the low-noise backgrounds in both instantaneous images. The averages both highlight the extent of the jet sweep as well as steady features in the flow. 


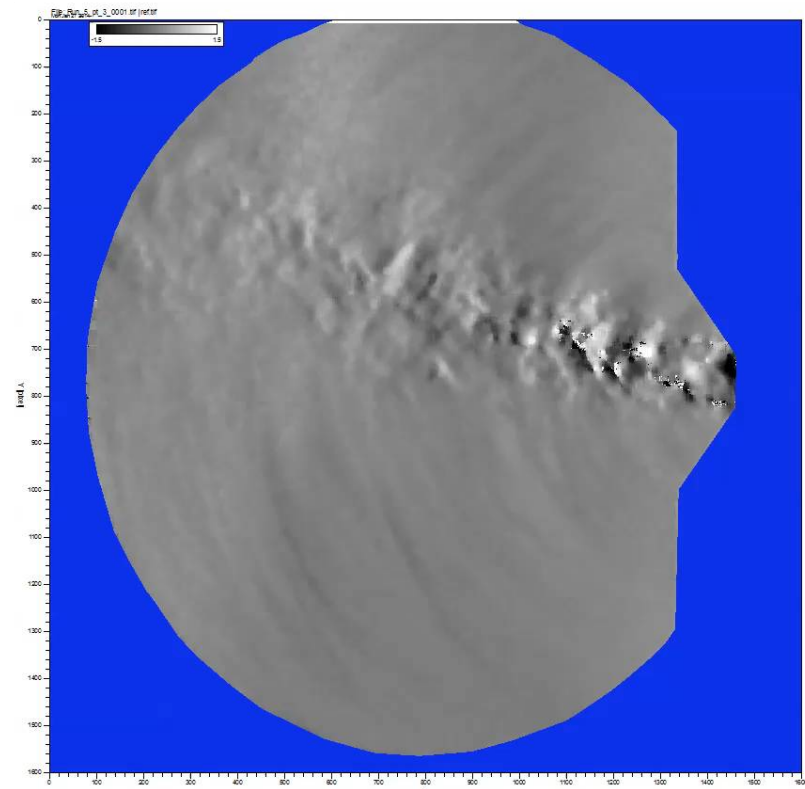

(a) $2 \mathrm{kHz}$ - Instantaneous dX

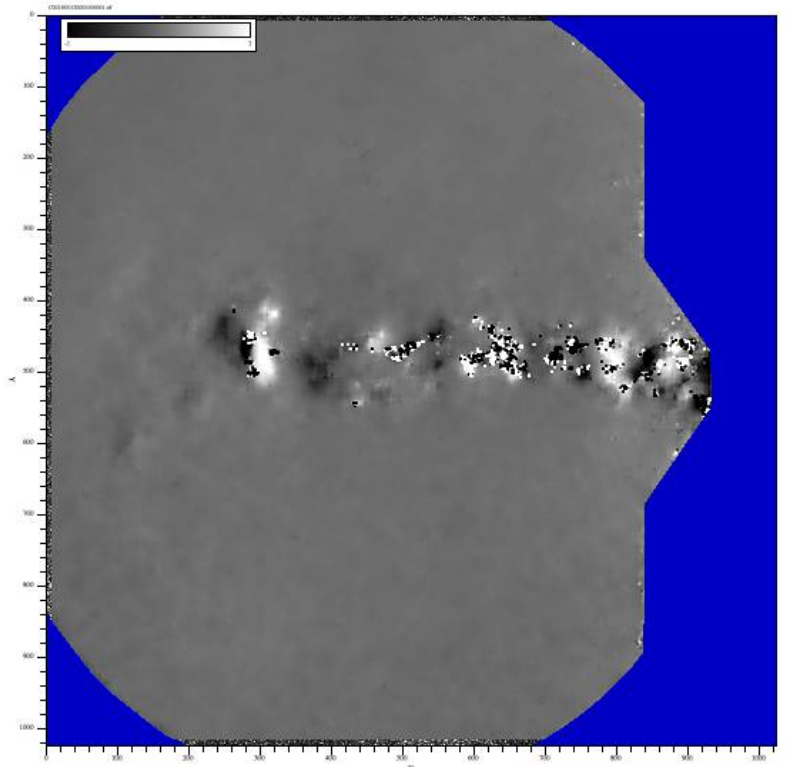

(c) $20 \mathrm{kHz}$ - Instantaneous dX

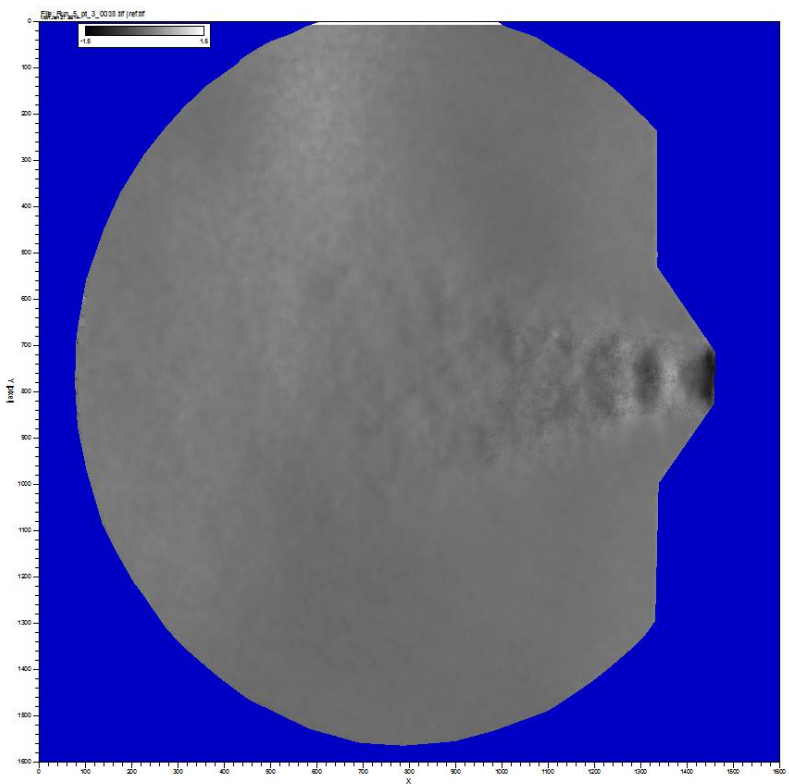

(b) $2 \mathrm{kHz}$ - Average dX

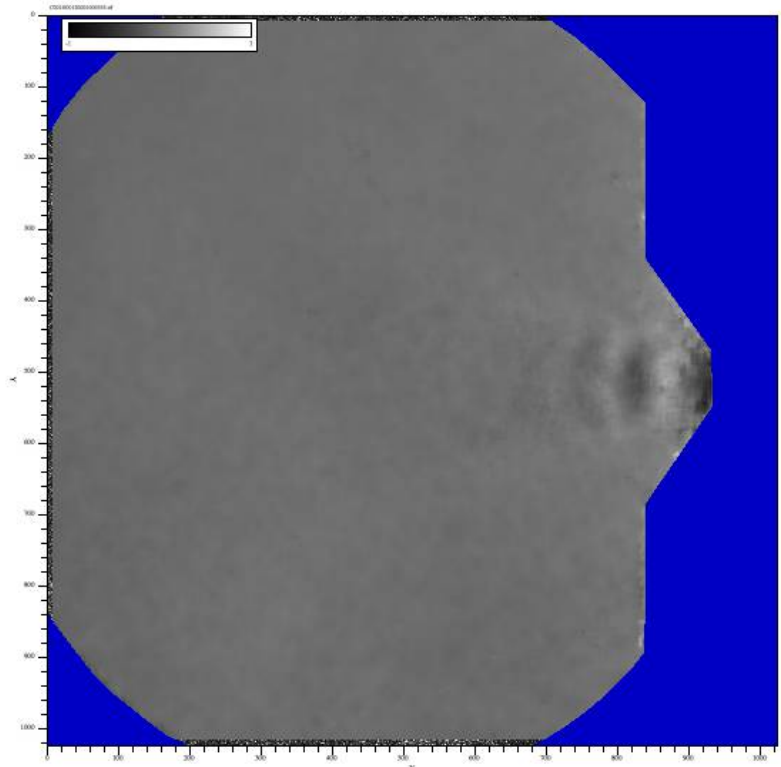

(d) $20 \mathrm{kHz}$-Average dX

Figure 5. Processed data at wind-off conditions and 38 psig actuator pressure. 


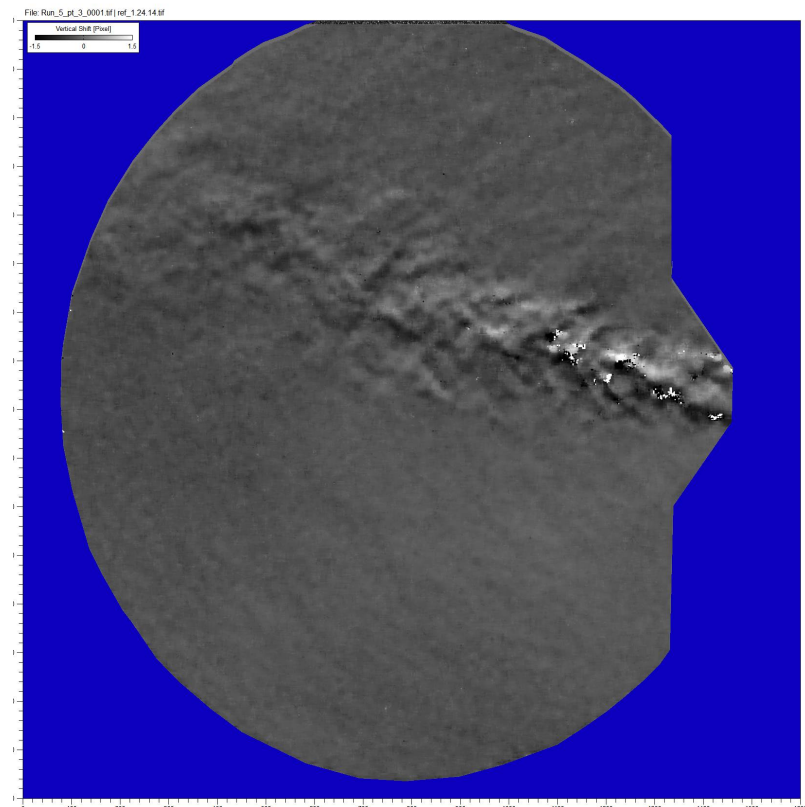

(a) $2 \mathrm{kHz}$ - Instantaneous dY

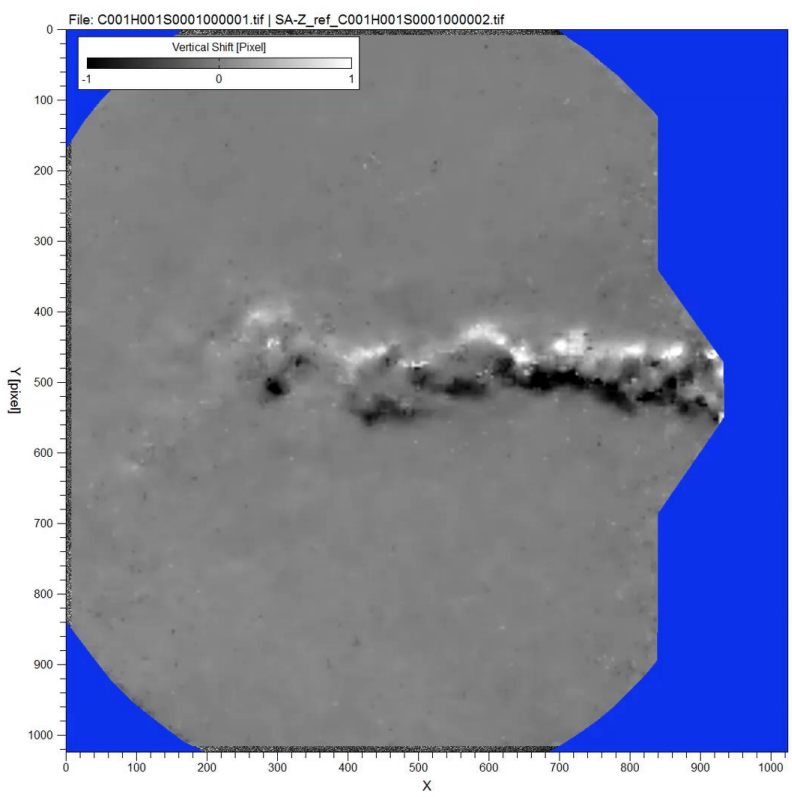

(c) $20 \mathrm{kHz}$ - Instantaneous dY

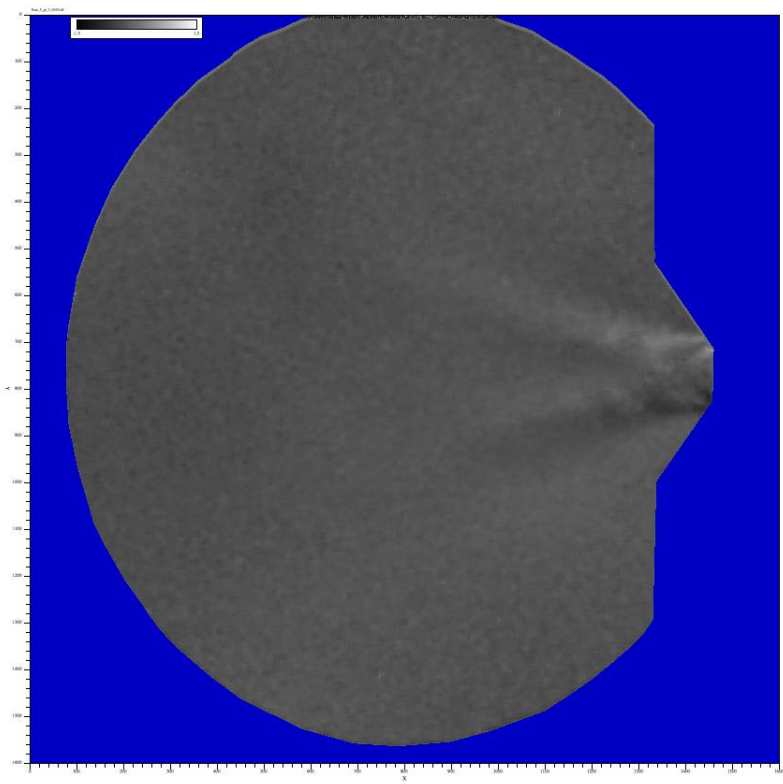

(b) $2 \mathrm{kHz}$ - Average dY

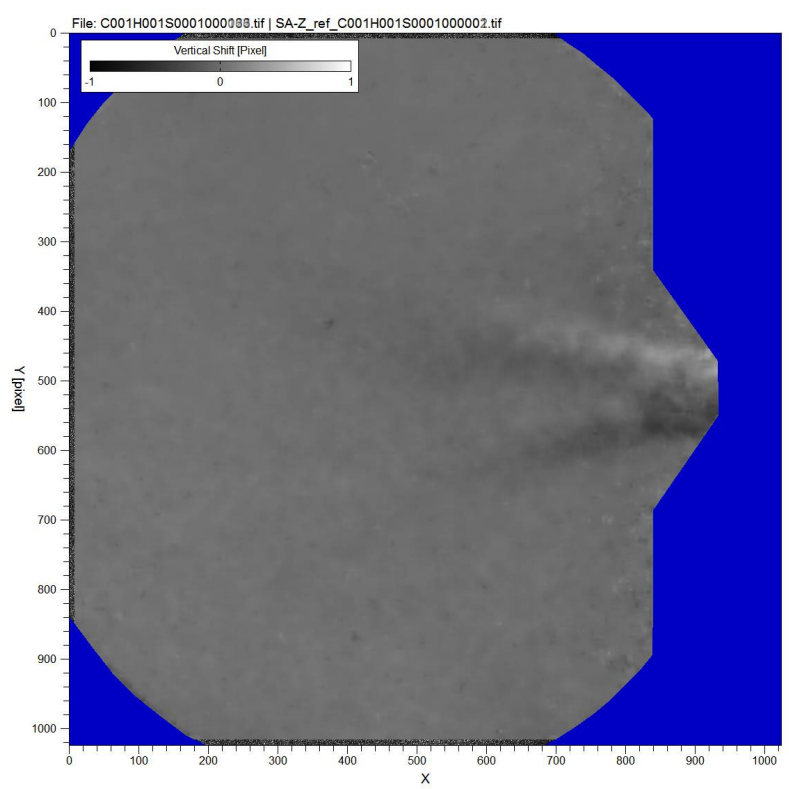

(d) $20 \mathrm{kHz}$-Average dY

Figure 6. Processed data at wind-off conditions and 38 psig actuator pressure. 


\section{CFD}

The Overflow CFD code (version 2.2f) was used to simulate the sweeping jet actuator at some of the flow conditions studied with RBOS. Overflow solves the compressible Navier-Stokes equations on a system of overset structured grids, using any of several spatial and temporal discretization algorithms. These simulations were done with a 5th-order WENO spatial scheme with a 2nd-order time-integration method driven by an SSOR inner solver. Several turbulence models are available in Overflow. These simulations were run with the SST model in the RANS-mode (Reynolds-Averaged Navier-Stokes) inside the fluidic actuator, and SST in the DDES-mode (Delayed detached eddy simulation) for the exterior flow. This choice of turbulence model was guided by comparisons of measured and simulated pressure histories near the throat of the nozzle. ${ }^{6}$ The CFD grid had on the order of 36 million points. The time step was $5 \times 10^{-6}$ seconds. The inner-iteration convergence of the integrated loads on the nozzle throat were monitored to guide in the choice of the time-integration settings.

Density gradients predicted by CFD are given in Figure 7. All results involve averaging over a $\sim 1 \mathrm{~mm}$ thick layer of the solution centered on the middle of the jet. (Data were recorded in this region for comparison to PIV measurements.) This spatial averaging is consistent with Schlerien line-of-sight integration, but it does not encompass the full thickness of the jet. Additional time averaging was done over an ensemble of time-realizations, as noted in the caption, which matches the RBOS data processing.

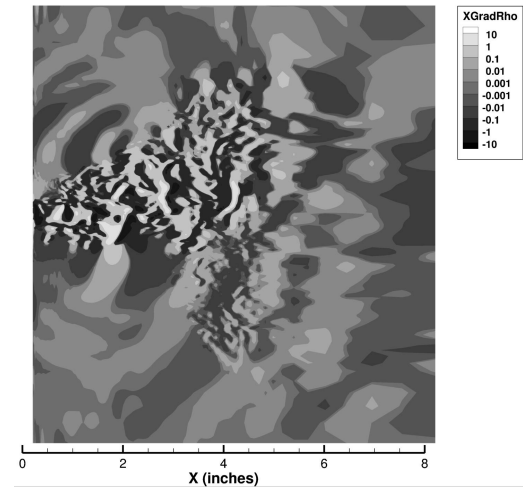

(a) Instantaneous $\mathrm{dX}$

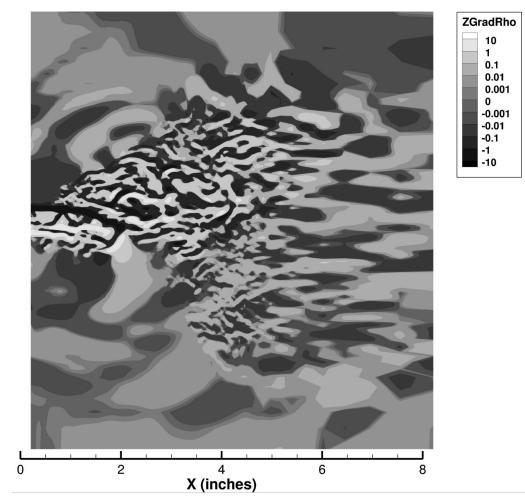

(d) Instantaneous $\mathrm{dY}$

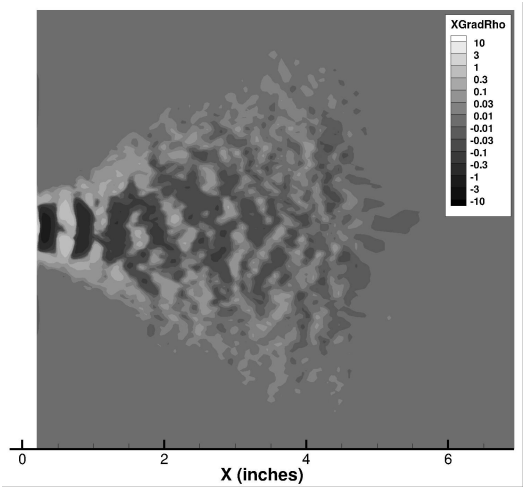

(b) $2 \mathrm{kHz}$ - Average dX

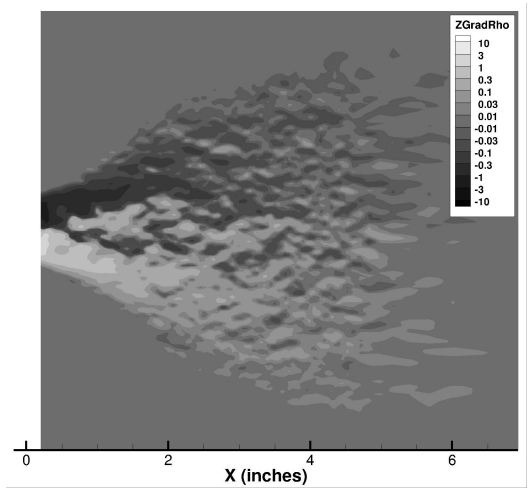

(e) $2 \mathrm{kHz}$-Average dY

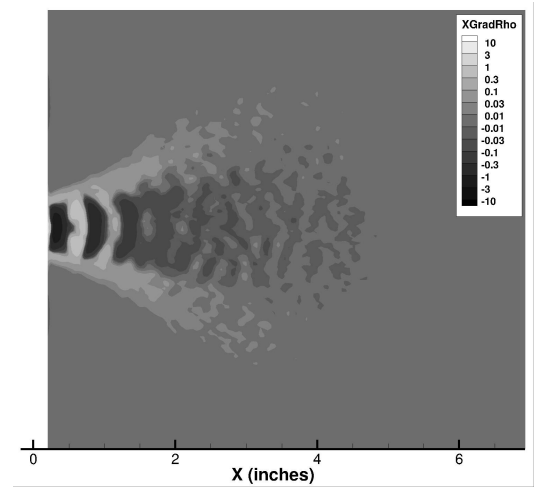

(c) $20 \mathrm{kHz}$ - Average dX

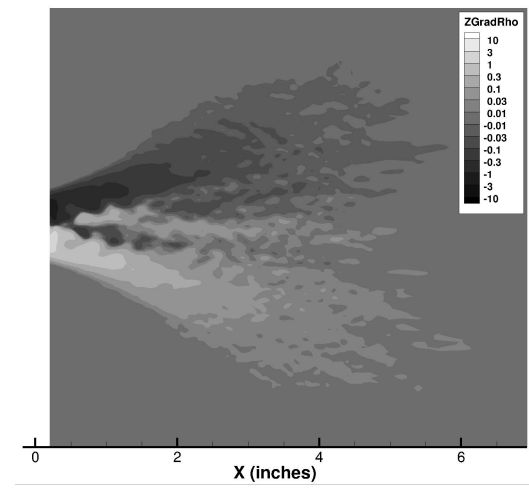

(f) $20 \mathrm{kHz}$-Average dY

Figure 7. CFD computed with Overflow.

\section{Discussion}

The RBOS data clearly capture the motion and structure of the jet for cases in which the tunnel is not running, and closely match the CFD simulations. However, for the wind on runs, tunnel vibration causes the retroreflector on the tunnel wall to shift, changing the speckle pattern. When the data is processed, the change in the speckle pattern causes the SNR of the background to change throughout the run and 
the visibility of the jet in the data changes accordingly. Stiffer backgrounds or another method of reducing vibration will be important in future work.

Future work will also include expansion of the laser RBOS technique to larger-scale tests.

\section{Conclusion}

Laser RBOS data were acquired on a single sweeping jet actuator embedded in an airfoil in the 48-by-32inch wind tunnel in the Fluid Mechanics Laboratory at NASA Ames Research Center. Data were acquired over a range of frequencies and sweeping jet pressures. The non-sinusoidal motion of the sweeping jet was successfully time-resolved at $20 \mathrm{kHz}$. The RBOS data clearly capture the motion and structure of the jet for cases in which the tunnel is not running, and closely match the CFD simulations. Laser RBOS produces very clean, low-noise backgrounds. It is best suited to low-vibration experiments, as vibration of the background causes the speckle pattern to shift which can overwhelm the signal from the density changes.

\section{Acknowledgments}

This work would not have been possible without funding from the NASA ERA program.

\section{References}

\footnotetext{
${ }^{1}$ Richard, H.; Raffel, M.; "Principle and applications of the background oriented schlieren (BOS) method." Measurement, Science and Technology, 12: 9, 1576-1585, 2001.

${ }^{2}$ Heineck, J. T.; Schairer, E. T.; Kushner, L. K; Walker,L. A.; "Retroreflective Background Oriented Schlieren (RBOS) As Applied to Full-Scale UH-60 Blade Tip Vortices", American Helicopter Society Aeromechanics Specialists' Conference, San Francisco, CA Jan. 20-22, 2010.

${ }^{3}$ Meier, A. H.; Roesgen, T. "Improved background oriented schlieren imaging using laser speckle illumination," Experiments in Fluids, Volume 54, Issue 6, pp.1-6, 2013.

${ }^{4}$ Koklu, M.; Melton, L. P.; "Sweeping Jet Actuator in a Quiescent Environment", AIAA 43rd Fluid Dynamics and Colocated Conferences. June 24-27, 2013, San Diego, CA.

${ }^{5}$ PIVview 3C Software Package, Version 3.5, PIVTEC GmbH, Gttingen, Germany.

$>6$ Lockard, D. P.; Choudhari, M. M.; Buning, P. G. ; "Grid Sensitivity Study for Slat Noise Simulations," 20th AIAA/CEAS Aeroacoustics Conference, 2014, 16-20 June 2014, Atlanta, GA. 10.2514/6.2014-2627(AIAA 2014-2627)
} 